\title{
Solving Singular Control from Optimal Switching
}

\author{
Xin Guo* $\quad$ Pascal Tomecek ${ }^{\dagger}$ \\ May 13, 2008
}

\begin{abstract}
This report summarizes some of our recent work (Guo and Tomecek $(2008 \mathrm{~b}, \mathrm{a})$ ) on a new theoretical connection between singular control of finite variation and optimal switching problems. This correspondence not only provides a novel method for analyzing multi-dimensional singular control problems, but also builds links among singular controls, Dynkin games, and sequential optimal stopping problems.
\end{abstract}

\section{Introduction}

In our recent work (Guo and Tomecek (2008b)), we established a generic theoretical connection between singular control and optimal switching problems: we defined a consistency property for collections of switching controls, and proved that there is an exact correspondence between the set of finite variation càglàd processes and the set of consistent collections of switching controls.

This correspondence allows one to analyze multi-dimensional control problem under a general setting for the regularity properties and the smooth fit principle directly: one can obtain an integral representation for the value function of a general class of singular control problem in terms of the values of corresponding optimal switching problems.

As a byproduct, we showed that the value of a Dynkin game can be represented as the difference between the values of two related switching problems, thereby linking the general reversible investment problem, the Dynkin game, and the optimal switching problem.

Continuing our analysis on singular control problems with possible non-smooth payoff functions, we (Guo and Tomecek (2008a)) analyzed a class of singular control problems for which value functions are not necessarily smooth. Necessary and sufficient conditions for the well-known smooth fit principle, along with the regularity of the value functions, are given. Explicit solutions for the optimal policy and for the value functions are provided. In

${ }^{*}$ Department of Industrial Engineering and Operations Research, UC Berkeley, CA 94720-1777, Phone (510) 642 3615, Email (xinguo@ieor . berkeley .edu). Generous support from OpenLink Fund at the Coleman Fung Risk Management Research Center at UC Berkeley is gratefully acknowledged.

${ }^{\dagger}$ J.P. Morgan, New York, email: pascal.i.tomecek@jpmorgan.com 
particular, when payoff functions satisfy the usual Inada conditions, the boundaries between action and no-action regions are smooth and strictly monotonic as postulated and exploited in the existing literature (Dixit and Pindyck (1994); Davis, Dempster, Sethi, and Vermes (1987); Kobila (1993); Abel and Eberly (1997); Øksendal (2000); Scheinkman and Zariphopoulou (2001); Merhi and Zervos (2007); Alvarez (2006)). Illustrative examples for both smooth and non-smooth cases are discussed to highlight the pitfall of solving singular control problems with a priori smoothness assumptions.

Previous work Singular control problems have been studied extensively in both the mathematics and economics, starting from the well-known monotone fuel follower problem, for which explicit solutions can be found in Bather and Chernoff (1967a,b); Beneš et al. (1980); Karatzas (1983) and Harrison and Taksar (1983). In mathematical economics, a typical (ir)reversible investment problem can be formulated as a singular control problem in which a company, by adjusting its production capacity through expansion and contraction according to market fluctuations, wishes to maximize its overall expected net profit over an infinite horizon. This problem has been investigated by numerous authors (See for instance Davis et al. (1987); Kobila (1993); Abel and Eberly (1997); Baldursson and Karatzas (1997); Øksendal (2000); Scheinkman and Zariphopoulou (2001); Wang (2003); Chiarolla and Haussmann (2005); Bank (2005); Guo and Pham (2005), and Merhi and Zervos (2007)). For a standard reference on irreversible investment, see Dixit and Pindyck (1994).

Our approach of connecting singular control problems and related optimal stopping problems dates back to the seminal paper of Bather and Chernoff (1967a), and has since been developed and applied to monotone singular control problems by Karatzas (1983, 1985); Karatzas and Shreve (1984, 1985, 1986); El Karoui and Karatzas (1988, 1989, 1991), and Baldursson and Karatzas (1997) ${ }^{1}$. Indeed, our integral representation theorem for the reversible investment problem is in part inspired by the elegant integration arguments of Baldursson and Karatzas (1997) for irreversible investment. Another closely related body of work is Boetius and Kohlmann (1998); Boetius (2001, 2003, 2005). However, the connections between the singular control problem, the entry-exit problem, and Dynkin's game in their works are established within the framework of forward backward stochastic differential equations and require a finite time horizon with the restrictive assumption that the control has only an additive affect on the diffusion.

Our contribution Compared to all previous works and approaches, the correspondence between singular controls and switching controls in our paper does not depend on the specific form of the control problem. Thus, our methodology may be applied to cases for which the underlying randomness is not necessarily captured by a diffusion and the payoff function is not necessarily smooth.

\footnotetext{
${ }^{1}$ Recently, Hamadène and Hassani (2006) observed that both the Dynkin game and the two regime optimal switching problem lead to BSDE's with two reflecting barriers.
} 


\section{Correspondence between Singular Controls and Switch- ing Controls}

The correspondence established in Guo and Tomecek (2008b) is analogous to the well-known correspondence between a non-decreasing, $\mathbb{F}$-adapted, càglàd singular control $\left(\xi_{t}\right)_{t \geq 0}$ and a collection of stopping times $\left(\tau^{\xi}(z)\right)_{z \in \mathbb{R}}$, given by

$$
\tau^{\xi}(z)=\inf \left\{t \geq 0: \xi_{t}>z\right\}, \quad \text { and } \quad \xi_{t}=\sup \left\{z \in \mathbb{R}: \tau^{\xi}(z)<t\right\}
$$

\subsection{Definitions}

Let $(\Omega, \mathcal{F}, P)$ be a complete probability space and $\mathbb{F}=\left\{\mathcal{F}_{t} ; 0 \leq t<\infty\right\}$ a filtration satisfying the usual hypotheses. Let $\mathcal{I} \subset \mathbb{R}$ be an open (possibly unbounded) interval, and $\overline{\mathcal{I}}$ be its closure.

Definition 2.1. Given $y \in \overline{\mathcal{I}}$, an admissible singular control is a pair $\left(\xi_{t}^{+}, \xi_{t}^{-}\right)_{t \geq 0}$ of $\mathbb{F}$ adapted, non-decreasing càglàd processes such that $\xi^{+}(0)=\xi^{-}(0)=0, Y_{t}:=y+\xi_{t}^{+}-\xi_{t}^{-} \in$ $\overline{\mathcal{I}}, \forall t \in[0, \infty)$, and $d \xi^{+}, d \xi^{-}$are supported on disjoint subsets.

We denote here $\mathcal{A}_{y}$ to be the set of admissible strategies corresponding to an initial capacity level of $y$.

Since $d \xi^{+}, d \xi^{-}$are supported on disjoint subsets, $\xi^{+}$and $\xi^{-}$are the positive and negative variation of $Y$, respectively. By the uniqueness of the variation decomposition, there is a one-to-one correspondence between strategies $\left(\xi^{+}, \xi^{-}\right) \in \mathcal{A}_{y}$ and $\mathbb{F}$-adapted càglàd finite variation processes $Y$ with $Y_{0}=y$ and $Y_{t} \in \overline{\mathcal{I}}$ for all $t$.

Throughout the paper, $\left(Y_{t}\right)_{t \geq 0}$ is a finite variation control process with $Y_{0}=y$.

Definition 2.2. A switching control $\alpha=\left(\tau_{n}, \kappa_{n}\right)_{n \geq 0}$ consists of an increasing sequence of stopping times $\left(\tau_{n}\right)_{n \geq 0}$ and a sequence of new regime values $\left(\kappa_{n}\right)_{n \geq 0}$ that are assumed immediately after each stopping time.

When there are only two distinct regimes, an optimal switching problem is often referred to as the starting and stopping problem (Brekke and Øksendal (1994); Hamadène and Jeanblanc (2004), etc.) or the entry and exit problem (Boetius (2003); Duckworth and Zervos (2000), etc.). Following convention, we label the two regimes 0 and 1.

Definition 2.3. A switching control $\alpha=\left(\tau_{n}, \kappa_{n}\right)_{n \geq 0}$ is admissible if the following hold almost surely: $\tau_{0}=0, \tau_{n+1}>\tau_{n}$ for $n \geq 1, \tau_{n} \rightarrow \infty$, and for all $n \geq 0, \kappa_{n} \in\{0,1\}$ is $\mathcal{F}_{\tau_{n}}$ measurable, with $\kappa_{n}=\kappa_{0}$ for even $n$ and $\kappa_{n}=1-\kappa_{0}$ for odd $n$.

Alternatively, an admissible switching control has a more mathematically convenient representation given by its regime indicator function. 
Proposition 2.4. There is a one-to-one correspondence between admissible switching controls and the regime indicator function $I_{t}(\omega)$, which is an $\mathbb{F}$-adapted càglàd process of finite variation, so that $I_{t}(\omega): \Omega \times[0, \infty) \rightarrow\{0,1\}$, with

$$
I_{t}:=\sum_{n=0}^{\infty} \kappa_{n} 1_{\left\{\tau_{n}<t \leq \tau_{n+1}\right\}}, \quad I_{0}=\kappa_{0} .
$$

Definition 2.5. Let $y \in \overline{\mathcal{I}}$ be given, and for each $z \in \mathcal{I}$, let $\alpha(z)=\left(\tau_{n}(z), \kappa_{n}(z)\right)_{n \geq 0}$ be a switching control. The collection $(\alpha(z))_{z \in \mathcal{I}}$ is consistent if

$$
\begin{aligned}
& \alpha(z) \text { is admissible for Lebesgue-almost every } z \in \mathcal{I}, \\
& I_{0}(z):=\kappa_{0}(z)=1_{\{z \leq y\}}, \text { for Lebesgue-almost every } z \in \mathcal{I},
\end{aligned}
$$

and for all $t<\infty$,

$$
\begin{aligned}
& \int_{\mathcal{I}}\left(I_{t}^{+}(z)+I_{t}^{-}(z)\right) d z<\infty, \text { almost surely, and } \\
& I_{t}(z) \text { is decreasing in } z \text { for } \mathbb{P} \otimes d z \text {-almost every }(\omega, z) .
\end{aligned}
$$

Here $I_{t}(z), I_{t}^{+}(z)$ and $I_{t}^{-}(z)$ are $I_{t}=\kappa_{0}+I_{t}^{+}-I_{t}^{-}$, and $I_{t}^{+}\left(I_{t}^{-}\right)$is the positive (negative) variation of the corresponding regime indicator function such that

$$
I_{t}^{+}:=\sum_{n>0, \kappa_{n}=1}^{\infty} 1_{\left\{\tau_{n}<t\right\}}, \quad I_{0}^{+}=0 \quad \text { and } \quad I_{t}^{-}:=\sum_{n>0, \kappa_{n}=0}^{\infty} 1_{\left\{\tau_{n}<t\right\}}, \quad I_{0}^{-}=0 .
$$

For $I_{t}(z)$ to be decreasing in $z$ for $\mathbb{P} \otimes d z$-almost every $(\omega, z)$, it means there exists a set $E \subset \Omega \times \overline{\mathcal{I}}$ such that $\mathbb{P} \otimes d z(E)=0$ and if $\left(\omega, z_{0}\right),\left(\omega, z_{1}\right) \in(\Omega \times \overline{\mathcal{I}}) \backslash E$ with $z_{0} \leq z_{1}$, then $I_{t}\left(\omega, z_{0}\right) \geq I_{t}\left(\omega, z_{1}\right)$.

\subsection{Bijection}

The bijection between the singular control and the switching control was established based on a relatively old result in analysis (Evans and Gariepy, 1992, Theorem 5.5.1).

Proposition 2.6 (From Singular Controls to Switching Controls). Given $\left(\xi^{+}, \xi^{-}\right) \in \mathcal{A}_{y}$, define a switching control $\alpha(z)=\left(\tau_{n}(z), \kappa_{n}(z)\right)_{n \geq 0}$ for each $z \in \mathcal{I}$ through the regime indicator function $I_{t}(z):=\lim _{s \uparrow t} 1_{\left\{Y_{s}>z\right\}}$. Then, the resulting collection $(\alpha(z))_{z \in \mathcal{I}}$ of switching controls is consistent.

Proposition 2.7 (From Switching Controls to Singular Controls). Given $y \in \overline{\mathcal{I}}$ and $a$ consistent collection of switching controls $(\alpha(z))_{z \in \mathcal{I}}$, define two processes $\xi^{+}$and $\xi^{-}$by setting $\xi_{0}^{+}=0, \xi_{0}^{-}=0$, and for $t>0: \xi_{t}^{+}:=\int_{\mathcal{I}} I_{t}^{+}(z) d z, \quad \xi_{t}^{-}:=\int_{\mathcal{I}} I_{t}^{-}(z) d z$. Then

1. The pair $\left(\xi^{+}, \xi^{-}\right) \in \mathcal{A}_{y}$ is an admissible singular control, 
2. Up to indistinguishability,

$$
Y_{t}=y+\int_{y}^{\infty} I_{t}(z) 1_{\{z \in \mathcal{I}\}} d z+\int_{-\infty}^{y}\left(I_{t}(z)-1\right) 1_{\{z \in \mathcal{I}\}} d z, \quad \text { and }
$$

3. For all t, we almost surely have

$$
Y_{t}=\operatorname{ess} \sup \left\{z \in \mathcal{I}: I_{t}(z)=1\right\}=\operatorname{ess} \inf \left\{z \in \mathcal{I}: I_{t}(z)=0\right\},
$$

where $\operatorname{ess} \sup \emptyset:=\inf \mathcal{I}$ and $\operatorname{ess} \inf \emptyset:=\sup \mathcal{I}$.

Proposition 2.8 (One-to-One Mapping). The mapping from consistent collections of switching controls to singular controls defined by Proposition 2.7 is one-to-one.

Theorem 2.9 (Bijection). The mappings in Propositions 2.6 and 2.7 define a bijection between admissible singular controls $\left(\xi^{+}, \xi^{-}\right) \in \mathcal{A}_{y}$ and consistent collections of switching controls (up to equivalence).

Given this correspondence, we shall use the following terminology in the sequel. Given a singular control $\left(\xi^{+}, \xi^{-}\right) \in \mathcal{A}_{y}$, the corresponding collection of switching controls $(\alpha(z))_{z \in \mathcal{I}}$ refers to the one defined in Proposition 2.6; given a consistent collection of switching controls, the corresponding singular control refers to that in Proposition 2.7.

\subsection{Change of Variable Formula}

With the bijection established in Theorem 2.9, we established a change of variable formula for integration with respect to the variation of a singular control.

Proposition 2.10. Let $\left(\xi^{+}, \xi^{-}\right) \in \mathcal{A}_{y}$ be an admissible singular control and $(\alpha(z))_{z \in \mathcal{I}}$ be the corresponding collection of switching controls. For every càdlàg process $g: \Omega \times[0, \infty] \rightarrow$ $[0, \infty)$ with $g(\infty) \equiv 0$,

$$
\begin{aligned}
\int_{[0, \infty)} g(t) d \xi_{t}^{+} & =\int_{\mathcal{I}} \sum_{\substack{n>0 \\
\kappa n=1}} g\left(\tau_{n}(z)\right) d z, \quad \text { a.s., } \\
\text { and } \int_{[0, \infty)} g(t) d \xi_{t}^{-} & =\int_{\mathcal{I}} \sum_{\substack{n>0 \\
\kappa n=0}} g\left(\tau_{n}(z)\right) d z, \quad \text { a.s. }
\end{aligned}
$$

In particular, when $Y$ is non-decreasing (i.e. $\xi^{-} \equiv 0$ ), $\overline{\mathcal{I}}=[0, \infty)$ and $y \geq 0$, we have $\tau_{n}(z) \equiv 0$ for all $n>1$, and for $n=1$ when $z \leq y$. In this case, our change of variable formula reduces to the one for monotone controls in Baldursson and Karatzas (1997), after adjusting for notational differences,

$$
\int_{[0, \infty)} g(t) d \xi_{t}^{+}=\int_{y}^{\infty} g\left(\tau_{1}(z)\right) d z .
$$




\section{Application I: Analysis of a Class of Singular Control Problems}

Having established the correspondence between singular controls and consistent collections of switching controls, we showed how this theory can be applied to analyzing singular control problems.

\subsection{The Singular Control Problem}

Consider the following class of singular control problems from economics named reversible investment problem: a company adjusts its reversible production capacity (or investment) level by proper controls of expansion and contraction in the presence of a stochastic economic environment. The net profit of such an investment depends on the running production function of the actual capacity, the economic uncertainty such as price or demand for the product, the benefits of contraction (e.g. via spinning off part of the business), and the cost of expanding and reducing the capital. The company's objective is to maximize the expected profit over an infinite time horizon by controlling expansion and contraction.

More specifically, the instantaneous operating profit of the company is a function of the production capacity and random variables representing the uncertain economic environment:

$$
\Pi(\omega, t, z): \Omega \times[0, \infty) \times \overline{\mathcal{I}} \rightarrow \mathbb{R}
$$

The unit cost of increasing the capacity at time $t$ is $\gamma_{+}(\omega, t): \Omega \times[0, \infty) \rightarrow \mathbb{R}$, and the unit cost of decreasing capacity is $\gamma_{-}(\omega, t): \Omega \times[0, \infty) \rightarrow \mathbb{R}$, where both $\gamma_{+}$and $\gamma_{-}$are adapted to $\mathbb{F}^{2}$

The control of the production capacity $Y_{t}$ is represented by a pair $\left(\xi_{t}^{+}, \xi_{t}^{-}\right)_{t \geq 0}$ of $\mathbb{F}$-adapted, non-decreasing càglàd processes such that

$$
\begin{aligned}
& \xi^{+}(0)=\xi^{-}(0)=0, \\
& Y_{t}=y+\xi_{t}^{+}-\xi_{t}^{-} \in \overline{\mathcal{I}}, \quad \forall t \in[0, \infty) .
\end{aligned}
$$

Here, $\xi_{t}^{+}$and $\xi_{t}^{-}$represent the cumulative expansion and reduction of capital until time $t$ respectively. We say the policy $\left(\xi^{+}, \xi^{-}\right)$is integrable if the integrability condition is satisfied for the initial capacity level $y$. That is,

$$
\mathbb{E}\left[\int_{0}^{\infty}\left|\Pi\left(t, Y_{t}\right)\right| d t+\int_{[0, \infty)}\left|\gamma_{+}(t)\right| d \xi_{t}^{+}+\int_{[0, \infty)}\left|\gamma_{-}(t)\right| d \xi_{t}^{-}\right]<\infty
$$

We denote $\mathcal{A}_{y}^{\prime} \subset \mathcal{A}_{y}$ as the set of integrable strategies.

\footnotetext{
${ }^{2}$ When there is no risk of ambiguity, we suppress the dependence of the profit and cost functions on $\omega$, writing $\Pi(t, z), \gamma_{+}(t)$ and $\gamma_{-}(t)$
} 
Faced with these profit and cost functions, the company must choose an investment strategy of capacity expansion and reduction which produces the following expected payoff over an infinite horizon:

$$
J\left(y, \xi^{+}, \xi^{-}\right):=\mathbb{E}\left[\int_{0}^{\infty} \Pi\left(t, Y_{t}\right) d t-\int_{[0, \infty)} \gamma_{+}(t) d \xi_{t}^{+}-\int_{[0, \infty)} \gamma_{-}(t) d \xi_{t}^{-}\right] .
$$

The objective is to maximize over all integrable policies $\left(\xi^{+}, \xi^{-}\right) \in \mathcal{A}_{y}^{\prime}$. Accordingly, the value function is defined as:

$$
V(y):=\sup _{\left(\xi^{+}, \xi^{-}\right) \in \mathcal{A}_{y}^{\prime}} J\left(y, \xi^{+}, \xi^{-}\right)
$$

Note that for any $y \in \overline{\mathcal{I}}, \mathcal{A}_{y}^{\prime}$ is not empty, as the expected profit of not investing at all (i.e. $\left.\xi^{+} \equiv 0 \equiv \xi^{-}\right)$is finite and is given by

$$
R(y):=J(y, 0,0)=\mathbb{E}\left[\int_{0}^{\infty} \Pi(t, y) d t\right] .
$$

\section{Standing assumptions}

A1. $\Pi$ is concave in $y$ and continuous at the boundary of $\mathcal{I}$, so that for $y_{1}<y_{2} \in \overline{\mathcal{I}}$,

$$
\Pi\left(t, y_{2}\right)-\Pi\left(t, y_{1}\right):=\int_{y_{1}}^{y_{2}} \pi(t, z) d z
$$

where $\pi$ is decreasing in $z$ a.s. and adapted to $\mathbb{F}$. Furthermore,

$$
\begin{aligned}
& \mathbb{E}\left[\int_{0}^{\infty}|\Pi(t, z)| d t\right]<\infty, \quad \forall z \in \overline{\mathcal{I}}, \\
& \mathbb{E}\left[\int_{0}^{\infty}|\pi(t, z)| d t\right]<\infty, \quad \forall z \in \mathcal{I} .
\end{aligned}
$$

This assumption implies that the value function is well defined and although it may take values of $+\infty$, it is never $-\infty$ since $V(y) \geq R(y)>-\infty$ by (14).

A2. $\gamma_{+}$and $\gamma_{-}$are adapted to $\mathbb{F}, \gamma^{ \pm}(\infty):=0$ and

$$
\gamma_{+}(t)+\gamma_{-}(t)>0, \quad \text { for all } t, \text { a.s. }
$$

This restriction eliminates the opportunity of making profit by simply switching regimes and immediately switching back.

A3. - If $\mathcal{I}$ is not bounded above, then $\gamma_{+}(t) \geq 0$ for all $t$ almost surely. And,

- if $\mathcal{I}$ is not bounded below, $\gamma_{-}(t) \geq 0$ for all $t$ almost surely.

This is to ensure that when the domain is unbounded, an arbitrarily large profit is not obtainable by arbitrarily large changes in the capacity level. 
A very special case for the above problem (11) is $\Pi(\omega, t, z)=e^{-\rho t}\left(X_{t}^{x}(\omega)\right)^{\lambda} z^{\beta}$, where the randomness in the economy is captured by the price process $X$ of the commodity, and $X$ is modelled by a geometric Brownian motion $d X_{t}^{x}=b X_{s}^{x} d t+\sqrt{2} \sigma X_{s}^{x} d W_{s}$, with $X_{0}=x>0$. The cost functions are $\gamma_{+}(\omega, t)=e^{-\rho t} K_{1}, \gamma_{-}(\omega, t)=e^{-\rho t} K_{0}$ for some constant $\rho>0, K_{0}, K_{1}$. This is an example first analyzed with explicit solutions by Merhi and Zervos (2007).

\subsection{Optimal Control from its Corresponding Optimal Switching Problem}

The key to using the connection between singular controls and switching controls to solve problem (11) in Section 3.1 is to write the payoff of this problem in terms of the payoffs of its corresponding optimal switching problems.

\subsubsection{Switching Controls from Singular Controls}

First, given the running profit and cost functions from the singular control problem (11), define a collection of optimal switching problems, indexed by $z \in \mathcal{I}$.

Definition 3.1. The switching cost process $\gamma: \Omega \times[0, \infty) \times\{0,1\} \rightarrow \mathbb{R}$ is given by

$$
\gamma(t, \kappa):=\gamma_{+}(t) 1_{\{\kappa=1\}}+\gamma_{-}(t) 1_{\{\kappa=0\}} .
$$

Here $\gamma(t, \kappa)$ represents the cost of switching to regime $\kappa$ at time $t$.

Next,

Proposition 3.2. Assume $\left(\xi^{+}, \xi^{-}\right) \in \mathcal{A}_{y}^{\prime}$. Let $(\alpha(z))_{z \in \mathcal{I}}$ be the corresponding consistent collection of switching controls with regime indicator functions $I(z)$, then

$$
J\left(y, \xi^{+}, \xi^{-}\right)-R(y)=\int_{y}^{\infty} m_{+}(z, \alpha(z)) 1_{\{z \in \mathcal{I}\}} d z+\int_{-\infty}^{y} m_{-}(z, \alpha(z)) 1_{\{z \in \mathcal{I}\}} d z .
$$

Where

$$
\begin{aligned}
m_{+}(z, \alpha) & :=\mathbb{E}\left[\int_{0}^{\infty} \pi(t, z) I_{t} d t-\sum_{n=1}^{\infty} \gamma\left(\tau_{n}, \kappa_{n}\right)\right] \in(-\infty, \infty) \\
\text { and } m_{-}(z, \alpha) & :=\mathbb{E}\left[\int_{0}^{\infty}-\pi(t, z)\left(1-I_{t}\right) d t-\sum_{n=1}^{\infty} \gamma\left(\tau_{n}, \kappa_{n}\right)\right] \in(-\infty, \infty)
\end{aligned}
$$

Here $m_{+}(z, \alpha), m_{-}(z, \alpha)$ are two expected payoffs for the switching controls for each $z \in \mathcal{I}$ and $\alpha \in \mathcal{B}$, with $\kappa_{0}=k \in\{0,1\}$. 


\subsubsection{Representation Theorem}

Now, for each $z \in \mathcal{I}$, the optimal switching control problem is to maximize the expected payoff over possible switching controls $\alpha \in \mathcal{B}$ such that $\kappa_{0}=k \in\{0,1\}$. This leads to the value functions given by

$$
\begin{aligned}
& m_{+}^{*}(z, k):=\sup _{\substack{\alpha \in \mathcal{B} \\
\kappa_{0}=k}} m_{+}(z, \alpha), \\
& m_{-}^{*}(z, k):=\sup _{\substack{\alpha \in \mathcal{B} \\
\kappa_{0}=k}} m_{-}(z, \alpha),
\end{aligned}
$$

where $m_{+}(z, \alpha)$ and $m_{-}(z, \alpha)$ are given by (17) and (18).

In fact, these two value functions (19) and (20) are essentially the same as shown in the following lemma.

Lemma 3.3. The value functions $m_{+}^{*}(z, k)$ and $m_{-}^{*}(z, k)$ in (19) and (20) satisfy, for $k \in$ $\{0,1\}$,

$$
m_{+}^{*}(z, k)-m_{-}^{*}(z, k)=\mathbb{E}\left[\int_{0}^{\infty} \pi(t, z) d t\right] .
$$

In addition, for fixed $k \in\{0,1\}$, each switching control $\alpha \in \mathcal{B}$ that is optimal for (19) will also be optimal for (20) and vice versa.

Theorem 3.4 (Representation). Fix $y \in \overline{\mathcal{I}}$, let $V(y)$ and $R(y)$ be given from $(11), m_{+}^{*}(z, k)$ and $m_{-}^{*}(z, k)$ be given by (19) and (20), and $\left(\hat{\xi}^{j+}, \hat{\xi}^{j-}\right) \in \mathcal{A}_{y}$ be the corresponding singular control as per Proposition 2.7. Assume there is a sequence of consistent collections of switching controls $\left(\alpha_{j}(z)\right)_{z \in \mathbb{R}}$ so that as $j \rightarrow \infty$,

$$
\begin{gathered}
\int_{y}^{\infty} m_{+}\left(z, \alpha_{j}(z)\right) 1_{\{z \in \mathcal{I}\}} d z+\int_{-\infty}^{y} m_{-}\left(z, \alpha_{j}(z)\right) 1_{\{z \in \mathcal{I}\}} d z \\
\rightarrow \int_{y}^{\infty} m_{+}^{*}(z, 0) 1_{\{z \in \mathcal{I}\}} d z+\int_{-\infty}^{y} m_{-}^{*}(z, 1) 1_{\{z \in \mathcal{I}\}} d z
\end{gathered}
$$

Assume also $\left(\hat{\xi}^{j+}, \hat{\xi}^{j-}\right) \in \mathcal{A}_{y}^{\prime}$ for all $j$. Then,

$$
V(y)-R(y)=\int_{y}^{\infty} m_{+}^{*}(z, 0) 1_{\{z \in \mathcal{I}\}} d z+\int_{-\infty}^{y} m_{-}^{*}(z, 1) 1_{\{z \in \mathcal{I}\}} d z .
$$

Moreover, with stronger assumptions, one can further establish the existence of an optimal control strategy.

\section{Assumption 3.5.}

1. [Existence of consistent controls] Fix $y \in \overline{\mathcal{I}}$ and let $m_{+}^{*}(z, k)$ and $m_{-}^{*}(z, k)$ be given by (19) and (20). For almost all $z \in \mathcal{I}$, there exists an optimal admissible switching control $\alpha(z) \in \mathcal{B}$ such that

$$
\begin{aligned}
m_{+}^{*}(z, 0)=m_{+}(z, \alpha(z)), & \text { for } z>y, \\
\text { and, } \quad & m_{+}^{*}(z, 1)=m_{+}(z, \alpha(z)), \quad \text { for } z \leq y .
\end{aligned}
$$

Furthermore, the collection $(\alpha(z))_{z \in \mathbb{R}}$ is consistent. 
2. [Integrability of singular control] Let $\left(\hat{\xi}^{+}, \hat{\xi}^{-}\right) \in \mathcal{A}_{y}$ be the corresponding singular control as per Proposition 2.7, then $\left(\hat{\xi}^{+}, \hat{\xi}^{-}\right) \in \mathcal{A}_{y}^{\prime}$.

Theorem 3.6 (Representation and Existence). Under Assumption 3.5, the Representation Theorem 3.4 holds. Moreover, the strategy $\left(\hat{\xi}^{+}, \hat{\xi}^{-}\right)$is optimal.

Although establishing simpler conditions for the consistency of the switching controls requires more structure for the control problem, the equally technical integrability assumption on the singular controls can be reduced to easily verifiable ones when $\mathcal{I}$ is bounded. These extra assumptions are in line with some of those in Merhi and Zervos (2007).

Theorem 3.7 (Sufficient Condition for Integrability). Let $\mathcal{I}$ be bounded, assume 3.5.1 and let $\left(\hat{\xi}^{+}, \hat{\xi}^{-}\right)$be the corresponding singular control as per Proposition 2.7. Furthermore, suppose

1. $\sup _{0 \leq t \leq T} \sup _{z \in \mathcal{I}}|\Pi(\omega, t, z)|<\infty$, almost surely, for all $T>0$,

2. $\quad \lim \sup _{T \rightarrow \infty} \mathbb{E}\left[\left|\gamma_{+}(T)\right|+\left|\gamma_{-}(T)\right|\right]<\infty$, and

3. For every strategy $\left(\xi^{+}, \xi^{-}\right) \in \mathcal{A}_{y}$, either $\left(\xi^{+}, \xi^{-}\right) \in \mathcal{A}_{y}^{\prime}$; Or, there exists an $\mathbb{F}$-adapted process $Z$ such that $U . \leq Z$. almost surely, $\mathbb{E}\left[\left|Z_{T}\right|\right]<\infty$ for all $T \geq 0$, and $\lim \sup _{T \rightarrow \infty} \mathbb{E}\left[Z_{T}\right]=-\infty$, where

$$
U_{T}\left(y, \xi^{+}, \xi^{-}\right):=\int_{0}^{T} \Pi\left(t, Y_{t}\right) d t-\int_{[0, T)} \gamma_{+}(t) d \xi_{t}^{+}-\int_{[0, T)} \gamma_{-}(t) d \xi_{t}^{-} .
$$

Then $\left(\hat{\xi}^{+}, \hat{\xi}^{-}\right) \in \mathcal{A}_{y}^{\prime}$. Hence Assumption 3.5 holds, yielding Theorem 3.6.

Note that when $\mathcal{I}$ is unbounded, integrable consistent controls may not exist under these extra conditions. Nevertheless,

Corollary 3.8. If $\mathcal{I}$ is unbounded, the assumptions of Theorem 3.7 yield Theorem 3.4.

\subsection{Regularity of the Value Function and Dynkin's Game}

Based on the representation theorem, we provided conditions under which the value function of the switching controls is not only continuous, but also continuously differentiable.

Theorem 3.9 (Regularity). Suppose that for some open interval $\mathcal{J} \subset \mathcal{I}$ and any $y \in \mathcal{J}$,

$$
\lim _{z \rightarrow y} \mathbb{E}\left[\int_{0}^{\infty}|\pi(t, z)-\pi(t, y)| d t\right]=0 .
$$

Suppose also that on $\mathcal{J}$, the value function has the representation

$$
V(y)-R(y)=\int_{y}^{\infty} m_{+}^{*}(z, 0) 1_{\{z \in \mathcal{I}\}} d z+\int_{-\infty}^{y} m_{-}^{*}(z, 1) 1_{\{z \in \mathcal{I}\}} d z .
$$

Then $V$ is $C^{1}$ on $\mathcal{J}$. And for any $y \in \mathcal{J}$,

$$
V^{\prime}(y)=\mathbb{E}\left[\int_{0}^{\infty} \pi(t, y) d t\right]+m_{-}^{*}(y, 1)-m_{+}^{*}(y, 0)=m_{+}^{*}(y, 1)-m_{+}^{*}(y, 0) .
$$


Note that our result generalizes previous ones of Karatzas and Wang (2001) and Boetius (2001, 2003, 2005) on the differentiability of the value function for the (ir)reversible investment problem. Another major difference is that the derivative in their work is in terms of the value of a Dynkin game, whereas the derivative here is the difference between the value functions of an optimal switching problem. In fact, our regularity results demonstrate that optimal switching problems provide a "missing link" between Dynkin games and singular control problems.

\subsubsection{Dynkin Games}

A Dynkin game is a game of timing between two players, whom we call MAX and MIN, following Boetius (2005). We fix some level $z \in \mathcal{I}$. While the game is in progress, MIN pays MAX at rate $\pi(t, z)$ and the game ends when one player chooses to stop. Thus, MAX and MIN each chooses strategies on when to exit the game (the stopping times $\sigma_{-}$and $\sigma_{+}$ respectively). The player to exit first receives an amount from her opponent equal to $\gamma_{-}\left(\sigma_{-}\right)$ if MAX exits first, and $\gamma_{+}\left(\sigma_{+}\right)$if MIN exits first. If both players exit at the same time, we treat it as though MIN exited first. Furthermore, each player may choose never to exit, i.e. $\sigma=\infty$. MAX chooses her strategy $\sigma_{-}$to maximize her payoff, and MIN chooses $\sigma_{+}$in order to minimize MAX's payoff.

This game is formally described below. To ensure that the payoff of the game is well defined, we assume in this section that for every stopping time $\sigma, \mathbb{E}\left[\left|\gamma_{-}(\sigma)\right|\right]<\infty$ and $\mathbb{E}\left[\left|\gamma_{+}(\sigma)\right|\right]<\infty$

Definition 3.10. Given $z \in \mathcal{I}$ and $\mathbb{F}$-stopping times $\sigma_{-}$and $\sigma_{+}$, the payoff of the Dynkin game is

$$
D\left(\sigma_{-}, \sigma_{+} ; z\right)=\int_{0}^{\sigma_{-} \wedge \sigma_{+}} \pi(t, z) d t+\gamma_{+}\left(\sigma_{+}\right) 1_{\left\{\sigma_{+} \leq \sigma_{-}\right\}}-\gamma_{-}\left(\sigma_{-}\right) 1_{\left\{\sigma_{-}<\sigma_{+}\right\}} .
$$

The game has a value if

$$
\sup _{\sigma_{-}} \inf _{\sigma_{+}} \mathbb{E}\left[D\left(\sigma_{-}, \sigma_{+} ; z\right)\right]=\inf _{\sigma_{+}} \sup _{\sigma_{-}} \mathbb{E}\left[D\left(\sigma_{-}, \sigma_{+} ; z\right)\right]
$$

we have,

Theorem 3.11. Given any $z \in \mathcal{I}$ such that conditions (15) and (16) hold, the value of the Dynkin game exists, and is equal to

$$
m_{+}^{*}(z, 1)-m_{+}^{*}(z, 0)=\sup _{\sigma_{+}} \inf _{\sigma_{-}} \mathbb{E}\left[D\left(\sigma_{-}, \sigma_{+} ; z\right)\right]=\inf _{\sigma_{-}} \sup _{\sigma_{+}} \mathbb{E}\left[D\left(\sigma_{-}, \sigma_{+} ; z\right)\right] .
$$

Furthermore,

Corollary 3.12. If (15) and (16) hold and $\pi(t, z)$ is decreasing in $z$, then $m_{+}^{*}(z, 1)-m_{+}^{*}(z, 0)$ is decreasing in $z$. 
That is, when the marginal payoff is decreasing in the capacity level $z$, the added benefit of being invested in the project at level $z$ is also decreasing in $z$. The economic interpretation is that there are decreasing returns to scale.

Note that for simplicity, we considered only infinite-horizon Dynkin game with no terminal payoff. With a slight modification, our arguments can be adapted for the finite horizon case.

\section{Explicit Solutions and Smooth Fit Principle}

To further analyze regularity properties and establish necessary and sufficient conditions for the smooth fit principle, we (Guo and Tomecek (2008a)) studied the following specific problem:

$$
V(x, y):=\sup _{\left(\xi^{+}, \xi^{-}\right) \in \mathcal{A}_{y}^{\prime}} J\left(x, y ; \xi^{+}, \xi^{-}\right),
$$

with

$$
J\left(x, y ; \xi^{+}, \xi^{-}\right):=\mathbb{E}\left[\int_{0}^{\infty} e^{-\rho t} H\left(Y_{t}\right) X_{t}^{x} d t-\int_{0}^{\infty} e^{-\rho t} K_{1} d \xi_{t}^{+}-\int_{0}^{\infty} e^{-\rho t} K_{0} d \xi_{t}^{-}\right],
$$

subject to

$$
\begin{aligned}
& Y_{t}:=y+\xi_{t}^{+}-\xi_{t}^{-}, \quad y \in[a, b], \\
& d X_{t}^{x}:=\mu X_{t}^{x} d t+\sqrt{2} \sigma X_{t}^{x} d W_{t}, \quad X_{0}:=x>0, \\
& H:[a, b] \rightarrow \mathbb{R} \text { is concave with } H(y)=H(a)+\int_{a}^{y} h(z) d z, \\
& K_{1}+K_{0}>0, \mu<\rho, \text { and (without loss of generality) } K_{1}>0 .
\end{aligned}
$$

The supremum is taken over all strategies $\left(\xi^{+}, \xi^{-}\right) \in \mathcal{A}_{y}^{\prime}$, where

$$
\begin{aligned}
\mathcal{A}_{y}^{\prime}:= & \left\{\left(\xi^{+}, \xi^{-}\right): \xi^{ \pm} \text {are left continuous, non-decreasing processes, } \xi_{0}^{ \pm}=0 ;\right. \\
& y+\xi_{t}^{+}-\xi_{t}^{-} \in[a, b] \\
& \left.\mathbb{E}\left[\int_{0}^{\infty} e^{-\rho t} d \xi_{t}^{+}+\int_{0}^{\infty} e^{-\rho t} d \xi_{t}^{-}\right]<\infty .\right\}
\end{aligned}
$$

We solved this problem completely and explicitly. (For readers' convenience, sketches of derivation are provided in the Appendix).

\subsection{Explicit Solutions}

\section{Theorem 4.1. [Value function]}

$$
V(x, y)=\eta H(a) x+\int_{a}^{y} v_{1}(x, z) d z+\int_{y}^{b} v_{0}(x, z) d z,
$$


where $v_{0}$ and $v_{1}$ are given explicitly based on $K_{0}$ :

Case I $\left(K_{0} \geq 0\right)$ :

1. For each $z \in(a, b)$ such that $h(z)=0: v_{0}(x, z)=v_{1}(x, z)=0$.

2. For each $z \in(a, b)$ such that $h(z)>0$ :

$$
\left\{\begin{array}{l}
v_{0}(x, z)= \begin{cases}A(z) x^{n}, & x<G(z), \\
\eta h(z) x-K_{1}, & x \geq G(z),\end{cases} \\
v_{1}(x, z)=\eta h(z) x,
\end{array}\right.
$$

where $G(z)=\nu h(z)^{-1}$, and $A(z)=\frac{K_{1}}{(n-1)}\left(\frac{h(z)}{\nu}\right)^{n}$, with $\nu=K_{1} \sigma^{2} n(1-m)$.

3. For each $z \in(a, b)$ such that $h(z)<0$ :

$$
\left\{\begin{array}{l}
v_{0}(x, z)=0, \\
v_{1}(x, z)= \begin{cases}B(z) x^{n}+\eta h(z) x, & x<F(z), \\
-K_{0}, & x \geq F(z),\end{cases}
\end{array}\right.
$$

where $F(z)=-\frac{\kappa}{h(z)}$, and $B(z)=\frac{K_{0}}{(n-1)} \kappa^{-n}\left(-\frac{h(z)}{\kappa}\right)^{n}$, with $\kappa=K_{0} \sigma^{2} n(1-m)$.

Case II $\left(K_{0}<0\right)$ :

1. For each $z \in(a, b)$ such that $h(z) \leq 0: v_{0}(x, z)=0, v_{1}(x, z)=-K_{0}$.

2. For each $z \in(a, b)$ such that $h(z)>0$ :

$$
\begin{aligned}
& v_{0}(x, z)= \begin{cases}A(z) x^{n}, & x<G(z), \\
B(z) x^{m}+\eta h(z) x-K_{1}, & x \geq G(z),\end{cases} \\
& v_{1}(x, z)= \begin{cases}A(z) x^{n}-K_{0}, & x \leq F(z), \\
B(z) x^{m}+\eta h(z) x, & x>F(z) .\end{cases}
\end{aligned}
$$

Here

$$
\begin{aligned}
& A(z)=\frac{h(z)^{n}}{(n-m) \nu^{n}}\left(\frac{\nu}{\sigma^{2}(n-1)}+m K_{1}\right)=\frac{h(z)^{n}}{(n-m) \kappa^{n}}\left(\frac{\kappa}{\sigma^{2}(n-1)}-m K_{0}\right) \\
& B(z)=\frac{-h(z)^{m}}{(n-m) \nu^{m}}\left(\frac{\nu}{\sigma^{2}(1-m)}-n K_{1}\right)=\frac{-h(z)^{m}}{(n-m) \kappa^{m}}\left(\frac{\kappa}{\sigma^{2}(1-m)}+n K_{0}\right) .
\end{aligned}
$$

The functions $F$ and $G$ are non-decreasing with

$$
F(z)=\frac{\kappa}{h(z)} \quad \text { and } \quad G(z)=\frac{\nu}{h(z)}
$$


where $\kappa<\nu$ are the unique solutions to

$$
\begin{aligned}
\frac{1}{1-m}\left[\nu^{1-m}-\kappa^{1-m}\right] & =-\frac{\rho}{m}\left[K_{1} \nu^{-m}+K_{0} \kappa^{-m}\right], \\
\frac{1}{n-1}\left[\nu^{1-n}-\kappa^{1-n}\right] & =\frac{\rho}{n}\left[K_{1} \nu^{-n}+K_{0} \kappa^{-n}\right] .
\end{aligned}
$$

Here, $m<0<1<n$ are the roots of $\sigma^{2} x^{2}+\left(\mu-\sigma^{2}\right) x-\rho=0$ such that

$$
m, n=\frac{-\left(\mu-\sigma^{2}\right) \pm \sqrt{\left(\mu-\sigma^{2}\right)^{2}+4 \sigma^{2} \rho}}{2 \sigma^{2}}
$$

and

$$
\eta:=\frac{1}{\rho-\mu}=\frac{-m n}{(n-1)(1-m) \rho}=\frac{1}{\sigma^{2}(n-1)(1-m)} .
$$

Theorem 4.2. [Optimal control] The optimal singular control $\left(\hat{\xi}^{+}, \hat{\xi}^{-}\right) \in \mathcal{A}_{y}^{\prime}$ exits. For each $z \in(a, b)$, the optimal control is described in terms of $F(z)$ and $G(z)$ from Theorem 4.1 such that

- (Case I, $\left.K_{0} \geq 0\right)$ : For $z$ such that $h(z)>0$, it is optimal to invest in the project past level $z$ when $X_{t}^{x} \in[G(z), \infty)$, and never disinvest. When $h(z)<0$, it is optimal to disinvest below level $z$ when $X_{t}^{x} \in[F(z), \infty)$, and it is never optimal to invest. When $h(z)=0$, it is optimal to neither invest nor disinvest (i.e. $F(z)=\infty=G(z)$ ).

- (Case II, $\left.K_{0}<0\right)$ : For z such that $h(z)>0$, it is optimal to invest in the project past level $z$ when $X_{t}^{x} \in[G(z), \infty)$, and to disinvest below level $z$ when $X_{t}^{x} \in(0, F(z)]$. For $z$ such that $h(z) \leq 0$, it is always optimal to disinvest.

Theorem 4.3. [Optimally controlled process] The resulting optimal control process $\hat{Y}_{t}$ is give by:

Case I: (up to indistinguishability) for $t>0$,

- If $h\left(y^{+}\right)>0$ then $\hat{Y}_{t}=\max \left\{G^{\rightarrow}\left(M_{t}\right), y\right\}$,

- If $h(y+)=0$ or $h(y-)=0$ then $\hat{Y}_{t}=y$,

- If $h(y-)<0$ then $\hat{Y}_{t}=\min \left\{F^{\rightarrow}\left(M_{t}\right), y\right\}$.

Here $M_{t}=\max \left\{X_{s}^{x}: s \in[0, t]\right\}$, and $F^{\rightarrow}$ and $G^{\rightarrow}$ are respectively the left-continuous inverses of $F$ (non-increasing) and $G$ (non-decreasing).

Case II: (up to indistinguishability) for $t>0$,

$$
\hat{Y}_{t}= \begin{cases}G^{\rightarrow}\left(M_{t}^{0}\right) \vee y, & \text { on }\left\{t \leq S_{1}\right\}, \\ F^{\leftarrow}\left(m_{t}^{n}\right) \wedge \hat{Y}_{S_{n}}, & \text { on }\left\{S_{n}<t \leq T_{n}\right\}, \\ G^{\rightarrow}\left(M_{t}^{n}\right) \vee \hat{Y}_{T_{n}}, & \text { on }\left\{T_{n}<t \leq S_{n+1}\right\},\end{cases}
$$


and $\lim _{n \rightarrow \infty} S_{n}=\infty=\lim _{n \rightarrow \infty} T_{n}$ almost surely.

Here $F^{\leftarrow}(x)$ and $G^{\rightarrow}(x)$ are respectively the right continuous inverse of $F$ and the leftcontinuous inverse of $G$. Moreover, the stopping times $\left(S_{n}\right)$ and $\left(T_{n}\right)$ are given by

$$
\begin{array}{ll}
S_{1}=\inf \left\{t>0:\left(X_{t}^{x}, \hat{Y}_{t}\right) \in \mathcal{S}_{0}\right\}, & T_{1}=\inf \left\{t>S_{1}:\left(X_{t}^{x}, \hat{Y}_{t}\right) \in \mathcal{S}_{1}\right\}, \\
S_{n}=\inf \left\{t>T_{n-1}:\left(X_{t}^{x}, \hat{Y}_{t}\right) \in \mathcal{S}_{0}\right\}, & T_{n}=\inf \left\{t>S_{n}:\left(X_{t}^{x}, \hat{Y}_{t}\right) \in \mathcal{S}_{1}\right\} .
\end{array}
$$

Lastly, the processes $M_{t}^{n}, m_{t}^{n}$ are defined by $M_{t}^{0}=\max \left\{X_{t}^{x}: 0 \leq s \leq t\right\}$, and

$$
m_{t}^{n}=\min \left\{X_{t}^{x}: S_{n} \leq s \leq t\right\} 1_{\left\{S_{n} \leq t\right\}}, \quad M_{t}^{n}=\max \left\{X_{t}^{x}: T_{n} \leq s \leq t\right\} 1_{\left\{T_{n} \leq t\right\}} .
$$

\subsection{Smooth Fit Principle and Region Characterization}

Given the explicit solution, we further established the following regularity results, together with the region characterization. (For more discussions about the implication of these regularity results for the traditional HJB/Variational Inequality approach, interested readers are referred to the discussion section in Guo and Tomecek (2008a)).

Theorem 4.4. [Sufficient Conditions] $V(x, y)$ is $C^{1}$ in $x$ for all $(x, y) \in(0, \infty) \times[a, b]$, and

$$
\frac{\partial}{\partial x} V(x, y)=\eta H(a)+\int_{a}^{y} \frac{\partial}{\partial x} v_{1}(x, z) d z+\int_{y}^{b} \frac{\partial}{\partial x} v_{0}(x, z) d z .
$$

Moreover, if $H$ is $C^{1}$ on an open interval $\mathcal{J} \subset[a, b]$, then $V(x, y)$ is $C^{1}$ in $y$ on $(0, \infty) \times \mathcal{J}$; that is, $V(x, y)$ is $C^{1,1}$ on $(0, \infty) \times \mathcal{J}$.

Theorem 4.5. [Necessary and Sufficient Conditions for Smooth Fit] $V(x, y)$ is continuously differentiable in $x$ for all $(x, y) \in(0, \infty) \times[a, b] . V(x, y)$ is differentiable in $y$ at the point $(x, y)$ if and only if

$$
(x, y) \in\{(x, y) \in(0, \infty) \times(a, b): H \text { is differentiable at } y\} \cup \mathcal{S}_{0} \cup \mathcal{S}_{1},
$$

where $\mathcal{S}_{0}$ and $\mathcal{S}_{1}$ are given in Eq. (35). Alternatively, it is not differentiable in y at the point $(x, y)$ if and only if

$$
(x, y) \in\{(x, y) \in(0, \infty) \times(a, b): H \text { is not differentiable at } y\} \cap \mathcal{C} .
$$

Theorem 4.6. [Region characterization] Under the optimal singular control $\left(\hat{\xi}^{+}, \hat{\xi}^{-}\right) \in$ $\mathcal{A}_{y}^{\prime}$, define the corresponding investment $\left(\mathcal{S}_{1}\right)$, disinvestment $\left(\mathcal{S}_{0}\right)$, and continuation $(\mathcal{C})$ regions by

$$
\begin{cases}\mathcal{S}_{0}:= \begin{cases}\left\{(x, z) \in(0, \infty) \times[a, b]: x \geq \lim _{w \uparrow z} F(w)\right\}, & \text { if } K_{0} \geq 0 \text { (Case I), } \\ \left\{(x, z) \in(0, \infty) \times[a, b]: x \leq \lim _{w \uparrow z} F(w)\right\}, & \text { if } K_{0}<0 \text { (Case II), }\end{cases} \\ \mathcal{S}_{1}:=\left\{(x, z) \in(0, \infty) \times[a, b]: x \geq \lim _{w \downarrow z} G(w)\right\}, \\ \mathcal{C}:=(0, \infty) \times[a, b] \backslash\left(\mathcal{S}_{0} \cup \mathcal{S}_{1}\right) .\end{cases}
$$


Then, the action and continuation regions can be characterized as

$$
\left\{\begin{array}{l}
\mathcal{S}_{0}=\left\{(x, y) \in(0, \infty) \times[a, b]: V_{y}(x, y)=-K_{0}\right\} \\
\mathcal{S}_{1}=\left\{(x, y) \in(0, \infty) \times[a, b]: V_{y}(x, y)=K_{1}\right\} \\
\mathcal{C}=\left\{(x, y) \in(0, \infty) \times[a, b]: V_{y^{-}}(x, y)>-K_{0}, V_{y^{+}}(x, y)<K_{1}\right\}
\end{array}\right.
$$

\section{References}

Abel, A. B. and J. C. Eberly (1997). An exact solution for the investment and value of a firm facing uncertainty, adjustment costs, and irreversibility. J. Econom. Dynam. Control 21(45), 831-852.

Alvarez, L. H. (2006). A general theory of optimal capacity accumulation under price uncertainty and costly reversibility. Working Paper, Helsinki Center of Economic Research, Finland.

Baldursson, F. M. and I. Karatzas (1997). Irreversible investment and industry equilibrium. Finance and Stoch. 1(1), 69-89.

Bank, P. (2005). Optimal control under a dynamic fuel constraint. SIAM J. Control Optim. 44 (4), 1529-1541 (electronic).

Bather, J. and H. Chernoff (1967a). Sequential decisions in the control of a spaceship. In Proc. Fifth Berkeley Sympos. Mathematical Statistics and Probability (Berkeley, Calif., 1965/66), Vol. III: Physical Sciences, pp. 181-207. Berkeley, Calif.: Univ. California Press.

Bather, J. and H. Chernoff (1967b). Sequential decisions in the control of a spaceship (finite fuel). J. Appl. Probability 4, 584-604.

Beneš, V. E., L. A. Shepp, and H. S. Witsenhausen (1980). Some solvable stochastic control problems. Stochastics 4(1), 39-83.

Boetius, F. (2001). Bounded variation singular stochastic control and associated Dynkin game. In Mathematical finance (Konstanz, 2000), Trends Math., pp. 111-120. Basel: Birkhäuser.

Boetius, F. (2003). Singular Stochastic Control and its Relations to Dynkin game and Entryexit Problems. Ph.d. dissertation, Universität Konstanz, Konstanz, Germany.

Boetius, F. (2005). Bounded variation singular stochastic control and Dynkin game. SIAM J. Control Optim. 44(4), 1289-1321 (electronic).

Boetius, F. and M. Kohlmann (1998). Connections between optimal stopping and singular stochastic control. Stochastic Process. Appl. r7(2), 253-281. 
Brekke, K. A. and B. Øksendal (1994). Optimal switching in an economic activity under uncertainty. SIAM J. Control Optim. 32(4), 1021-1036.

Chiarolla, M. B. and U. G. Haussmann (2005). Explicit solution of a stochastic, irreversible investment problem and its moving threshold. Math. Oper. Res. 30(1), 91-108.

Davis, M. H. A., M. A. H. Dempster, S. P. Sethi, and D. Vermes (1987). Optimal capacity expansion under uncertainty. Adv. in Appl. Probab. 19(1), 156-176.

Dixit, A. K. and R. S. Pindyck (1994). Investment under Uncertainty. Princeton, NJ: Princeton University Press.

Duckworth, J. K. and M. Zervos (2000). An investment model with entry and exit decisions. J. Appl. Probab. 37(2), 547-559.

El Karoui, N. and I. Karatzas (1988). Probabilistic aspects of finite-fuel, reflected follower problems. Acta Appl. Math. $11(3), 223-258$.

El Karoui, N. and I. Karatzas (1989). Integration of the optimal risk in a stopping problem with absorption. In Séminaire de Probabilités, XXIII, Volume 1372 of Lecture Notes in Math., pp. 405-420. Berlin: Springer.

El Karoui, N. and I. Karatzas (1991). A new approach to the Skorohod problem, and its applications. Stochastics Stochastics Rep. 34(1-2), 57-82.

Evans, L. C. and R. F. Gariepy (1992). Measure Theory and Fine Properties of Functions. Studies in Advanced Mathematics. Boca Raton, FL: CRC Press.

Guo, X. and H. Pham (2005). Optimal partially reversible investment with entry decision and general production function. Stochastic Process. Appl. 115(5), 705-736.

Guo, X. and P. Tomecek (2008a). A class of singular control problems and the smooth fit principle. Preprint, UC at Berkeley.

Guo, X. and P. Tomecek (2008b). Connections between singular control and optimal switching. SIAM J. Control Optim. 47(1), 421-443.

Hamadène, S. and M. Hassani (2006). BSDEs with two reflecting barriers driven by a Brownian and a Poisson noise and related Dynkin game. Electron. J. Probab. 11, no. 5, 121-145 (electronic).

Hamadène, S. and M. Jeanblanc (2004). On the starting and stopping problem: Application in reversible investments. Working Paper, Université du Maine and Université d'Every Val d'Essonne, France.

Harrison, J. M. and M. I. Taksar (1983). Instantaneous control of Brownian motion. Math. Oper. Res. 8(3), 439-453. 
Karatzas, I. (1983). A class of singular stochastic control problems. Adv. in Appl. Probab. 15(2), 225-254.

Karatzas, I. (1985). Probabilistic aspects of finite-fuel stochastic control. Proc. Nat. Acad. Sci. U.S.A. 82(17), 5579-5581.

Karatzas, I. and S. E. Shreve (1984). Connections between optimal stopping and singular stochastic control. I. Monotone follower problems. SIAM J. Control Optim. 22(6), 856877.

Karatzas, I. and S. E. Shreve (1985). Connections between optimal stopping and singular stochastic control. II. Reflected follower problems. SIAM J. Control Optim. 23(3), 433451.

Karatzas, I. and S. E. Shreve (1986). Equivalent models for finite-fuel stochastic control. Stochastics 18(3-4), 245-276.

Karatzas, I. and H. Wang (2001). Connections between bounded variation control and dynkin games. In J. Menaldi, E. Rofman, and A. Sulem (Eds.), Optimal Control and Partial Differential Equations (Volume in honor of A. Bensoussan), pp. 363-373. Amsterdam: IOS Press.

Kobila, T. Ø. (1993). A class of solvable stochastic investment problems involving singular controls. Stochastics Stochastics Rep. 43(1-2), 29-63.

Ly Vath, V. and H. Pham (2007). Explicit solution to an optimal switching problem in the two-regime case. SIAM J. Control Optim., 395-426.

Merhi, A. and M. Zervos (2007). A model for reversible investment capacity expansion. SIAM J. Control Optim. To appear.

Øksendal, A. (2000). Irreversible investment problems. Finance Stoch. 4 (2), 223-250.

Scheinkman, J. A. and T. Zariphopoulou (2001). Optimal environmental management in the presence of irreversibilities. J. Econom. Theory 96(1-2), 180-207. Intertemporal equilibrium theory: indeterminacy, bifurcations, and stability.

Wang, H. (2003). Capacity expansion with exponential jump diffusion processes. Stoch. Stoch. Rep. 75(4), 259-274.

\section{Appendix: Derivation of Solutions in Section 4}

The problem is solved in several steps. And the key is the representation theorem: first define and solve the corresponding switching control problem, then establish the consistency properties of the optimal control and verifying integrability conditions of the corresponding singular controls. 
Step 1: Corresponding optimal switching problem. First, let $R(x, y):=J(x, y ; 0,0)$ be the no-action expected payoff. Then,

$$
\begin{aligned}
R(x, y) & :=\mathbb{E}\left[\int_{0}^{\infty} e^{-\rho t} H(y) X_{t}^{x} d t\right]=\eta H(y) x \\
r(x, y) & :=R_{y}(x, y)=\mathbb{E}\left[\int_{0}^{\infty} e^{-\rho t} h(y) X_{t}^{x} d t\right]=\eta h(y) x .
\end{aligned}
$$

One can check that Standing assumptions A1, A2, and A3, and assumptions in Theorem 3.7 hold for this problem.

Now, the following lemma can be verified.

\section{Lemma 5.1.}

$$
V(x, y)=\eta H(a) x+\int_{a}^{y} v_{1}(x, z) d z+\int_{y}^{b} v_{0}(x, z) d z,
$$

where $v_{0}$ and $v_{1}$ for the optimal switching problem defined by

$$
v_{k}(x, z):=m_{+}^{*}(x, z, k)=\sup _{\substack{\alpha \in \mathcal{B} \\ \kappa_{0}=k}} \mathbb{E}\left[\int_{0}^{\infty} e^{-\rho t} h(z) X_{t}^{x} I_{t} d t-\sum_{n=1}^{\infty} e^{-\rho \tau_{n}} K_{\kappa_{n}}\right] .
$$

Here, $\alpha=\left(\tau_{n}, \kappa_{n}\right)_{n \geq 0}$ is an admissible switching control so that almost surely $\tau_{0}=0, \tau_{n+1}>$ $\tau_{n}$ for $n \geq 1, \tau_{n} \rightarrow \infty$, and for all $n \geq 0, \kappa_{n} \in\{0,1\}$, with $\kappa_{n}=\kappa_{0}$ for $n$ even and $\kappa_{n}=1-\kappa_{0}$ for $n$ odd. $\mathcal{B}$ is the subset of admissible switching controls $\alpha=\left(\tau_{n}, \kappa_{n}\right)_{n \geq 0}$ such that $\left.\mathbb{E}\left[\sum_{n=1}^{\infty} e^{-\rho \tau_{n}}\right]<\infty\right\}$, and $I_{t}$ is the regime indicator function for any given $\alpha \in \mathcal{B}$ so that $I_{t}=\sum_{n=0}^{\infty} \kappa_{n} 1_{\left\{\tau_{n}<t \leq \tau_{n+1}\right\}}$.

Next, modifying the argument in Ly Vath and Pham (2007, Theorem 3.1), we obtain

Proposition 5.2. $v_{0}$ and $v_{1}$ are the unique viscosity solutions with linear growth condition to the following system of variational inequalities:

$$
\begin{aligned}
\min \left\{-\mathcal{L} v_{0}(x, z),\right. & \left.v_{0}(x, z)-v_{1}(x, z)+K_{1}\right\}=0, \\
\min \left\{-\mathcal{L} v_{1}(x, z)-h(z) x,\right. & \left.v_{1}(x, z)-v_{0}(x, z)+K_{0}\right\}=0,
\end{aligned}
$$

with boundary conditions $v_{0}\left(0^{+}, z\right)=0$ and $v_{1}\left(0^{+}, z\right)=\max \left\{-K_{0}, 0\right\}$. Here $\mathcal{L}$ is the generator of the diffusion $X^{x}$, killed at rate $\rho$, given by $\mathcal{L} u(x, z)=\sigma^{2} x^{2} u_{x x}(x, z)+\mu x u_{x}(x, z)-$ $\rho u(x, z)$.

Step 2: Consistent collection of optimal switching controls. Applying the results of Ly Vath and Pham (2007) for switching controls to Problem (40), we see that for any given $z \in(A, B)$ and $k \in\{0,1\}$, the value function $v_{k}(\cdot, z)$ is continuously differentiable. Moreover, for each $z \in(A, B)$, an optimal switching control exists, and can be described in 
terms of the switching regions: for each $z \in(A, B)$, there exist $0<F(z)<G(z)<\infty$ such that it is optimal to switch from regime 0 to regime 1 (to invest in the project at level $z$ ) when $X_{t}^{x} \in[G(z), \infty$ ), and to switch from regime 1 to regime 0 (disinvest at level $z$ ) when $X_{t}^{x} \in[0, F(z)]$.

Therefore, given any initial value $y \in[A, B]$ for the singular control problem, a collection of optimal switching controls can be defined as follows.

For $(x, z) \in \mathcal{X} \times(A, B)$, define the switching control $\hat{\alpha}(x, z)=\left(\hat{\tau}_{n}, \hat{\kappa}_{n}\right)_{n \geq 0}$, starting from $\hat{\tau}_{0}=0$ and $\kappa_{0}=1_{\{z \leq y\}}$ by setting $\hat{\kappa}_{n}:=1-\kappa_{n-1}$ for all $n \geq 1$ and

- if $\kappa_{n-1}=0, \hat{\tau}_{n}:=\inf \left\{t>\tau_{n-1}: X_{t}^{x} \geq G(z)\right\}$, or

- if $\kappa_{n-1}=1, \hat{\tau}_{n}:=\inf \left\{t>\tau_{n-1}: X_{t}^{x} \leq F(z)\right\}$.

Moreover, by the regularity of the value functions, we solve for $F(z)$ and $G(z)$ explicitly in our case, obtaining $F(z)=\kappa z^{\frac{1-\beta}{\lambda}}$ and $G(z)=\nu z^{\frac{1-\beta}{\lambda}}$ where $\kappa$ and $\nu$ are unique solutions to

$$
\begin{aligned}
\frac{\beta}{\lambda-m}\left[\nu^{\lambda-m}-\kappa^{\lambda-m}\right] & =-\frac{\rho}{m}\left[K_{1} \nu^{-m}+K_{0} \kappa^{-m}\right], \\
\frac{\beta}{n-\lambda}\left[\nu^{\lambda-n}-\kappa^{\lambda-n}\right] & =\frac{\rho}{n}\left[K_{1} \nu^{-n}+K_{0} \kappa^{-n}\right] .
\end{aligned}
$$

Here $m<0<n$, and $n, m=\frac{-\left(b-\sigma^{2}\right) \pm \sqrt{\left(b-\sigma^{2}\right)^{2}+4 \sigma^{2} r}}{2 \sigma^{2}}$.

Finally, by checking the appropriate integrability conditions, and by noting that $F$ and $G$ are increasing in $z$, it is not hard to verify that the above collection of optimal switching controls is consistent. (See Figure 1).

Step 3: Optimal singular control and value functions. By Proposition 2.7, this consistent collection of optimal switching control corresponds to an admissible singular control $\left(\hat{\xi}^{+}, \hat{\xi}^{-}\right) \in \mathcal{A}_{y}$. Moreover, since $\mathcal{I}$ is bounded, it is integrable following Theorem 3.7.

Put together, the investment region is given by $\{(x, z): x \geq G(z)\}$ and the disinvestment region by $\{(x, z): x \leq F(z)\}$. $Y_{t}$ is constant when $\left(X_{t}, Y_{t}\right)$ is in the wait region, given by $\{(x, z): F(z)<x<G(z)\}$. If $(x, y)$ is in the investment (or disinvestment) region, then a jump is exerted at time zero to make $Y_{0+}=G^{-1}(x)$ (or $Y_{0+}=F^{-1}(x)$ ).

Finally, by Lemma 3.3 and Theorem 3.6 the value function has the following representation

$$
\begin{aligned}
V(x, y) & =R(x, y)+\int_{y}^{B} v_{0}(x, z) d z+\int_{A}^{y}\left(v_{1}(x, z)-r(x, z)\right) d z \\
& =R(x, A)+\int_{y}^{B} v_{0}(x, z) d z+\int_{A}^{y} v_{1}(x, z) d z,
\end{aligned}
$$


(1) For fixed $z_{0}$ switching control

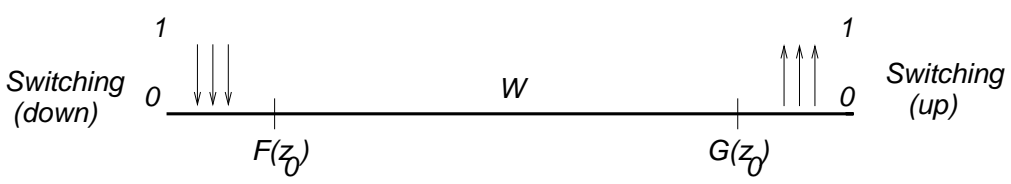

(2) For general z, consistent switching controls

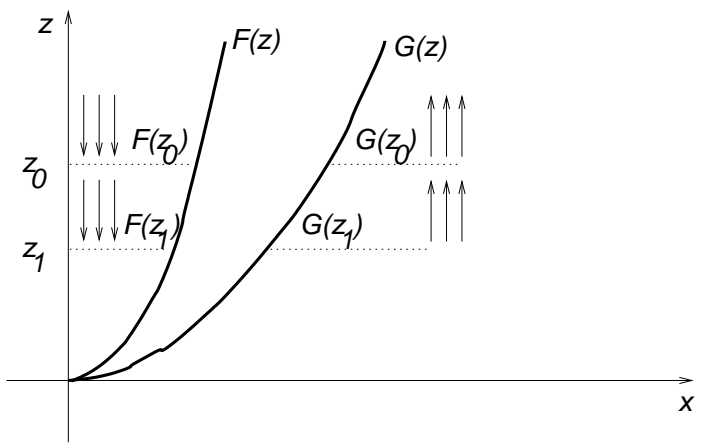

Figure 1: Illustration of optimal consistent switching control from optimal singular control where $v_{0}$ and $v_{1}$ are given by Ly Vath and Pham (2007, Theorem 4.2.3),

$$
\begin{aligned}
& v_{0}(x, z)= \begin{cases}A(z) x^{n}, & x<G(z), \\
B(z) x^{m}+r(x, z)-K_{1}, & x \geq G(z),\end{cases} \\
& v_{1}(x, z)= \begin{cases}A(z) x^{n}-K_{0}, & x \leq F(z), \\
B(z) x^{m}+r(x, z), & x>F(z) .\end{cases}
\end{aligned}
$$

Where $A(z)=\frac{\nu^{-n}}{n-m}\left(\frac{\beta \nu^{\lambda}}{\sigma^{2}(n-\lambda)}+m K_{1}\right) z^{\frac{-n(1-\beta)}{\lambda}}, B(z)=-\frac{\kappa^{-m}}{n-m}\left(\frac{\beta \kappa^{\lambda}}{\sigma^{2}(\lambda-m)}+n K_{0}\right) z^{\frac{-m(1-\beta)}{\lambda}}$. 